\title{
DNVF-Memorandum III - Methoden für die Versorgungsforschung, Teil 4 - Konzept und Methoden der organisationsbezogenen Versorgungsforschung
}

Kapitel 2 - Methodische Ansätze der organisationsbezogenen Versorgungsforschung: Zielgrößen, Datenquellen, Datenerhebung und Datenanalyse

DNVF-Memorandum III - Methods for Health Services Research, Part 4 - Concept and Methods for Organizational Health Services Research

Chapter 2 - Methodological Approaches for Organizational Health Services Research: Measures, Data Sources, Data Collection and Data Analysis

\section{(C) $(1) \odot$}

\section{Autoren}

Lars Rölker-Denker ${ }^{1}$, Christoph Kowalski², Lena Ansmann, 3, Ursula Hahn", 5, Antje Hammer ${ }^{6}$, Ramona Auer ${ }^{7}$, Tanja Kostuj ${ }^{8}$, Stefan Nöst ${ }^{9,} 10$

Institute

1 Fakultät für Medizin und Gesundheitswissenschaften, Department für Versorgungsforschung, Carl von Ossietzky Universität Oldenburg, Oldenburg

2 Deutsche Krebsgesellschaft e.V., Zertifizierung, Berlin

3 Humanwissenschaftliche Fakultät und Medizinische Fakultät, Institut für Medizinsoziologie, Versorgungsforschung und Rehabilitationswissenschaften, Universität zu Köln, Köln

4 Fakultät für Gesundheit der Universität Witten/ Herdecke, Institut für Medizinische Biometrie und Epidemiologie, Witten

5 OcuNet GmbH \& Co KG, Geschäftsführung, Düsseldorf

6 Institut für Patientensicherheit, Universitätsklinik Bonn, Bonn

7 Fachbereich Versorgungsmanagement, AOK BadenWürttemberg, Stuttgart

8 Klinik für Orthopädie und Unfallchirurgie, Klinikum Lippe, Lemgo

9 Studienfachbereich Gesundheit, Angewandte Gesundheits- und Pflegewissenschaften, Duale Hochschule Baden-Württemberg, Stuttgart

10 Abteilung Allgemeinmedizin und Versorgungsforschung, Universitatsklinikum Heidelberg, Heidelberg
Schlüsselwörter

Organisationsbezogene Versorgungsforschung, Versorgungsorganisation, Mixed methods, Memorandum

Key words organizational health services research, healthcare organization, mixed methods, memorandum

Bibliografie

DOI https://doi.org/10.1055/a-0862-0565

Gesundheitswesen 2019; 81: e72-e81

(c) Georg Thieme Verlag KG Stuttgart · New York

ISSN 0941-3790

Korrespondenzadresse

Prof. Lena Ansmann

Department für Versorgungsforschung

Abteilung Organisationsbezogene Versorgungsforschung

Carl von Ossietzky Universität Oldenburg

Ammerländer Heerstraße 140

26129 Oldenburg

lena.ansmann@uni-oldenburg.de 


\section{ZUSAMMENFASSUNG}

Das Forschungsfeld der organisationsbezogenen Versorgungsforschung ist in Deutschland noch relativ jung und von zunehmendem Interesse. Das Deutsche Netzwerk Versorgungsforschung e.V. (DNVF e.V.) hat im Jahr 2009 - getragen von den im DNVF organisierten Fachgesellschaften und Mitgliedern eine Vertiefung zu „Methoden der organisationsbezogenen Versorgungsforschung“ des Memorandums III, Teil 1 konsentiert [1]. Ausgehend von dieser Veröffentlichung und dem gegenwärtigen Bedeutungsgewinn des Forschungsfeldes ist eine Weiterentwicklung der konzeptionellen und methodischen Grundlage erforderlich. Die Neuauflage und Erweiterung der Veröffentlichung aus dem Jahr 2009 gliedert sich in 3 Kapitel: (1) Definition und Konzept der organisationsbezogenen Versorgungsforschung, (2) Methodische Ansätze der Organisationsbezogenen Versorgungsforschung: Zielgrößen, Datenquellen, Datenerhebung und Datenanalyse, (3) Methodische Ansätze zur Konzeption, Evaluation und Implementierung komplexer Interventionen in Versorgungsorganisationen. Die Herausarbeitung der methodischen Besonderheiten der organisationsbezogenen Versorgungsforschung - aufbauend auf den in Kapitel 1 dargestellten Besonderheiten des Forschungsgegenstandes - ist Ziel dieses zweiten Kapitels.

\section{ABSTRACT}

Organizational health services research is still a relatively young field of research in Germany which is of increasing interest. The German Network Health Services Research e.V. (DNVF e.V.) published in 2009 - supported by expert associations and individual members of the DNVF - a guide on "Methods for organizational health services research" of the Memorandum III, part 1 [1]. Originating from this publication and facilitated by the increasing relevance of the field, a necessity to refine the conceptual and methodological basis became evident. The update and extension of the publication from 2009 consists of 3 chapters: (1) Definition and concept of organizational health services research, (2) Methodological approaches in organizational health services research: indicators, data sources, data collection and data analysis, (3) Methodological approaches for the design, evaluation and implementation of complex interventions in healthcare organizations. The aim of the second chapter is to derive methodological requirements and characteristics of organizational health services research - based on the unique characteristics of the research field presented in chapter 1.

\section{Einleitung}

In der Versorgungsforschung wird der Organisationsbezug von Forschungsfragen und -daten häufig nicht gesehen. Umso wichtiger ist es, die Möglichkeiten der organisationsbezogenen Versorgungsforschung hier aufzuzeigen. Prämissen, auf die sich das Kapitel stützt und die die Relevanz der organisationsbezogenen Versorgungsforschung hervorheben, sollen vorab umrissen werden. Zum einen ist voranzustellen, dass es der organisationsbezogenen Versorgungsforschung - wie in Kapitel 1 [2] mit der Unterscheidung zwischen dem institutionellen und dem instrumentellen Organisationsbegriff beschrieben - nicht nur darum geht, zu identifizieren, welche Versorgungsorganisation welches Ergebnis hervorbringt. Es geht vielmehr darum, zu verstehen, warum dieses Ergebnis so zustande kommt. Dies bedeutet, dass Organisationsstrukturen und -prozesse, die Einfluss auf das Versorgungsergebnis haben, gleichermaßen zum Gegenstand der organisationsbezogenen Versorgungsforschung werden wie Unterschiede in der Ergebnisqualität zwischen den Versorgungsorganisationen selbst. Damit verbunden hat die organisationsbezogene Versorgungsforschung besonderes Interesse an dem Verstehen der Mechanismen, die Interventionen zugrunde liegen (sog. Black Box), die oftmals in die Routinen von Versorgungsorganisationen eingreifen. Da Studien der organisationsbezogenen Versorgungsforschung nicht in kontrollierbaren Umwelten stattfinden, sondern in unkontrollierbaren, dynamischen Umgebungen innerhalb komplexer Organisationen (siehe Kap. 3 [3]), ist zu betonen, dass es für die organisationsbezogene Versorgungsforschung nicht die eine passende Methodik oder das universelle Studiendesign gibt. Es wird ausdrücklich empfohlen, Studien an Theorien, die die Komplexität von Organisationen berücksichtigen (siehe Kap. 1 [2]) sowie an etablierten Forschungsrahmen (siehe Kap. 3 [3]) auszurichten.
Ziel dieses Kapitels ist es, auf Basis der Besonderheiten des Gegenstandes der organisationsbezogenen Versorgungsforschung zentrale Kategorien von Zielgrößen herauszuarbeiten und methodische Ansätze zu beschreiben, die der Komplexität des Gegenstandes gerecht werden. Darüber hinaus werden Datenquellen, Erhebungsmethoden sowie Auswertungsmethoden der organisationsbezogenen Versorgungsforschung dargestellt. Für detaillierte Empfehlungen zu Methoden der Evaluation und Implementierung von Interventionen in Versorgungsorganisationen sei auf Kapitel 3 [3] verwiesen.

\section{Zielgrößen}

Die letztlich maßgeblichen Zielgrößen von Studien der organisationsbezogenen Versorgungsforschung sind - wie generell in der Versorgungsforschung - die Outcomes auf Patientenebene ${ }^{1}$ im Sinne von Ergebnisqualität. Zuletzt wurden nach Schrappe und Pfaff [4] auch populationsbezogene Outcomes, wie Qualität und Wirtschaftlichkeit, als zentrale Zielgrößen definiert. Diese Outcome-Parameter sind nicht spezifisch für die organisationsbezogene Versorgungsforschung, sondern finden auch in Versorgungsforschungsstudien ohne unmittelbaren Organisationsbezug Verwendung und sind andernorts umfassend beschrieben (siehe Standards zur Definition patientenrelevanter Endpunkte [5,6] und das DNVF-Memorandum zur Qualitäts- und Patientensicherheitsforschung [7]). Die Besonderheiten von Versorgungsorganisationen machen es wie oben beschrieben notwendig, auch weitere Zielgrößen mit einzubeziehen, die im Folgenden erläutert werden und bereits an anderer Stelle anhand konkreter Studien aus Deutschland erläutert wurden [8].

1 Obwohl aus Gründen der besseren Lesbarkeit im Text die männliche Form gewählt wurde, beziehen sich alle Angaben auf alle Geschlechter. 
Eine Besonderheit von Versorgungsorganisationen leitet sich aus dem ordnungspolitischen Rahmen des deutschen Gesundheitssystems ab, das von vielfältigen marktförmigen und nichtmarktförmigen Steuerungsmechanismen geprägt ist (Korporatismus, staatliche Administration und Regulierung, Wettbewerb, u. a.). Diese makropolitischen Dynamiken haben direkt und indirekt Auswirkungen auf die Handlungs- und Entscheidungsprämissen in der Organisation und folglich auch auf das Outcome auf Patienten- und Populationsebene: Für das Gut Gesundheit gelten die zentralen Voraussetzungen des Marktmodells im Gesundheitssystem allerdings nur eingeschränkt. Im Gesundheitssystem herrscht eine Informationsasymmetrie zwischen Anbieter und Nachfrager (z. B. Arzt und Patient), der Preismechanismus versagt, die Elastizität der Nachfrage ist eher gering und gegebenenfalls angebotsinduziert. Diese ordnungspolitischen und ökonomischen Besonderheiten trugen begleitet durch weitere gesellschaftliche Entwicklungen, z. B. der Ausbildung der Medizin als Profession, christliche Traditionen, die berufsständige Organisation der Zünfte [9] - historisch betrachtet dazu bei, dass sich Strukturen und Prozesse in Versorgungsorganisationen herausgebildet haben, die von wettbewerblichen Märkten abweichen [10]. Damit geht einher, dass die organisationalen Kontextfaktoren sowie die Organisationen selbst aus einer Versorgungsforschungsperspektive als komplexe Entitäten betrachtet werden müssen. Studiendesigns sollten diese Komplexität insofern berücksichtigen, dass sowohl umweltbezogene als auch organisationale Faktoren unabhängig voneinander oder in Interaktion miteinander auf mehrere abhängige Variablen einwirken. Analysen sowie die Evaluation von Interventionen in Versorgungsorganisationen sollten aus diesem Grund typischer Weise nicht nur mit Blick auf patientenseitige oder populationsbezogene Outcomes durchgeführt werden. Vielmehr müssen neben diesen weitere vermittelnde Zielgrößen einbezogen werden [4]. Kontextfaktoren sind keine konstanten, kontrollierbaren Größen, aber „[...] der Kontext selbst [erhält] eine aktive Rolle beim Zustandekommen der Wirkung “ [4, S. 691]. Damit bekommen Organisationsstrukturen und -prozesse dieselbe Bedeutung wie der eigentliche Organisationszweck. Sie erscheinen nicht mehr nur als Mittel zum Zweck [11], sondern werden selbst Gegenstand der organisationsbezogenen Versorgungsforschung. Die von Schrappe und Pfaff vorgeschlagene aktualisierte Definition, dass Versorgungsforschung „ausgehend von der Patienten- und Populationsperspektive und vor dem Hintergrund komplexer Kontextbedingungen die Versorgungsstrukturen und -prozesse der Gesundheitsversorgung untersucht" [4, S. 691], greift diesen zentralen Aspekt unmittelbar auf. Auch das Throughput-Modell von Schrappe und Pfaff [4] transportiert diese Aussage und bildet damit einen wichtigen Referenzrahmen für die organisationsbezogene Versorgungsforschung.

\section{Kategorien des organisationalen Kontextes}

Die Organisationsstrukturen, die Organisationsprozesse und die Organisationskultur werden im Memorandum zu Methoden der organisationsbezogenen Versorgungsforschung aus 2009 [1] als zentrale Kategorien des organisationalen Kontextes definiert, die mit dem Outcome zusammenhängen ( $\triangleright$ Abb. 1). Neben der Analyse von Strukturen sowie Prozessen und der Evaluation von Struktur- und Prozessveränderungen innerhalb von Versorgungsorganisationen ist die Untersuchung der Organisationskultur eine zent-

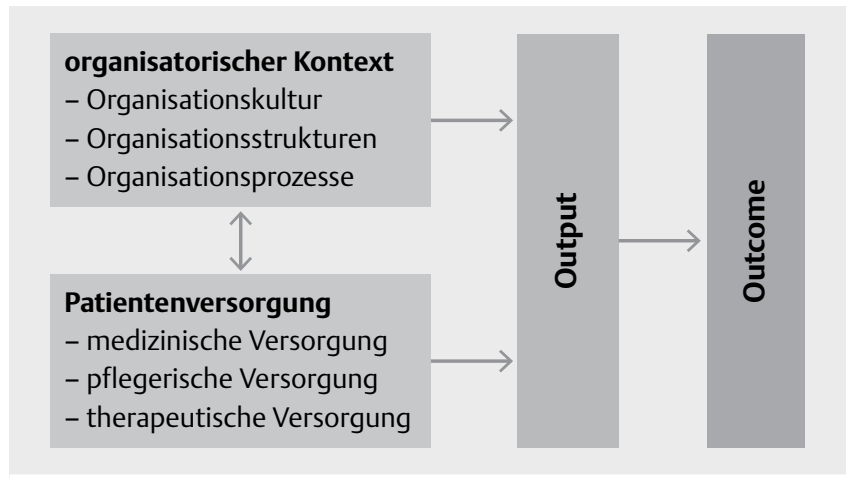

Abb. 1 Patientenversorgung und organisatorischer Kontext als Determinanten des Outputs und Outcomes (nach [1]).

rale Zielgröße der organisationsbezogenen Versorgungsforschung, die bisher noch wenig Aufmerksamkeit erhält [12]. Wenngleich Kultur mit Strukturen und Prozessen im Alltagshandeln untrennbar verbunden ist, ist es notwendig, sie von einer analytischen Perspektive aus zu trennen. Der Organisationspsychologe Edgar Schein beschreibt Kultur als Muster gemeinsamer Grundprämissen, das die Organisationsmitglieder bei der Bewältigung von Problemen unterstützt und durch interne Integration erlernt wird [13]. Die Kultur ist ein tief verankertes Gut einer Organisation, die nur langfristig und mit tiefgreifenden organisationalen Prozessen verändert werden kann [14, 15]. Die Organisationskultur bestimmt neben dem Organisationsziel maßgeblich die Entscheidungen aller Hierarchieebenen einer Organisation. Beispielsweise kann das Führungsverständnis Auswirkungen auf die Arbeitszufriedenheit und das Arbeitsverhalten der Professionals in der Organisation haben. Entsprechend wirken sich diese unmittelbar auf die Bereitschaft zur Umsetzung neuer Interventionen und damit auf das Outcome auf Patienten- und Populationsebene aus. Kultur wird in der organisationsbezogenen Versorgungsforschung bisher quantitativ, basierend auf Wahrnehmungen der Professionals erfasst. Übersichten und Empfehlungen zu geeigneten Erhebungsinstrumenten sind bereits einschlägig veröffentlicht $[12,16]$. Aus methodischer Perspektive erfordert ein tieferes Verständnis der Organisationskultur die Nutzung qualitativer Methoden [17]. Mit dem Ziel einer verstehenden Analyse der Organisationskultur sind rekonstruktive Verfahren der Sozialforschung angemessen $[18,19]$.

Um die hier beschriebenen Kategorien von Zielgrößen untersuchen zu können, wird ein methodischer Ansatz notwendig, der die Komplexität von Versorgungsorganisationen widerspiegelt.

\section{Methodenansatz der organisationsbezogenen Versorgungsforschung}

Versorgungsorganisationen, wie Krankenhäuser, Arztpraxen oder ambulante Pflegedienste, erbringen Versorgungsleistungen für Patienten sowie Populationen mit unterschiedlichem Bedarf und interagieren hierbei mit unterschiedlichen Akteuren sowie wechselnden Umwelten (vgl. Mikro-Meso-Makro Modell, Kap. 1 [2]). Während der traditionelle Ansatz der Versorgungsforschung die Probleme in den Blick nimmt, die bei der Umsetzung von individuellen Behandlungsmethoden unter Alltagsbedingungen entstehen (efficacy-effectiveness-gap), stellen aktuellere Definitionsversuche 
und Konzepte der Versorgungsforschung komplexe Interventionen auf Organisations- und Systemebene in den Vordergrund [4] und weisen dadurch die Komplexität selbst als Forschungsperspektive aus. Die organisationbezogene Versorgungsforschung erklärt dabei die organisationalen Bedingungen als intermediäre Zielgrößen der Patientenversorgung zum Forschungsgegenstand und beschreibt den Outcome unter Berücksichtigung der Ebene der Organisation (vgl. Kap. 1 [2]). Diese konzeptionelle Ausrichtung ist eng verknüpft mit der Entwicklung der Versorgungsforschung hin zur Improvement Science, die sich damit beschäftigt, wie und unter welchen Kontextbedingungen Verbesserungen und Veränderungen in der Versorgung am effektivsten funktionieren [20]. Die organisationsbezogene Versorgungsforschung bedarf daher der Anwendung angemessener Methoden, die je nach Forschungsfrage und -gegenstand in der Lage sind, (1.) die äußeren Rahmenbedingungen und Wechselwirkungen (Mikro-Meso-Makro Modell), (2.) die formalen und informalen Aspekte der Versorgungsorganisation (Organisationsstrukturen, Organisationsprozesse, Organisationskultur) sowie (3.) die Interaktion zwischen Organisationen verstehend zu rekonstruieren und modellieren sowie (4.) die Aus- und Wechselwirkungen von Versorgungsmaßnahmen auf oben genannte Zielgrößen unter Berücksichtigung dieser organisationalen Bedingungen zu erklären (vgl. Kap. 1 [2]). Davon ausgehend werden in der organisationsbezogenen Versorgungsforschung bevorzugt Forschungsdesigns gewählt, die nicht nur das Ergebnis eines Versorgungsprozesses evaluieren und bewerten, sondern gerade auch auf die Beschreibung der Mechanismen in der Black Box „Throughput“ zielen. Es geht somit in der organisationsbezogenen Versorgungsforschung nicht nur darum, zu identifizieren, welche Versorgungsorganisation welches Ergebnis hervorbringt, sondern es geht vielmehr darum, zu untersuchen, warum und wie dieses Ergebnis zustande kommt. Dazu müssen Studiendesigns und Methoden gewählt werden, die in der Lage sind, Versorgungsorganisationen zu verstehen, deskriptiv zu beschreiben und zu analysieren „wie sie sind“ [11] und nicht dem Rationalitätsparadigma folgend ,wie sie sein sollen“. Als Beispiel kann die Dokumentationspraxis in Krankenhäusern dienen, die angepasst wird, um die Anforderungen der externen Qualitätssicherung zu erfüllen, aber nicht zwangsläufig auch um die Qualität der Versorgung zu sichern oder steigern. Schließlich scheint es äußerst bedeutsam, relevante Determinanten des Zustandekommens eines Behandlungsergebnisses zu erkennen und zu verstehen, um zielgerichtet Interventionen gestalten, Organisationsentwicklung anstoßen und organisationbezogene Maßnahmen initiieren zu können [7, 11]. Um das notwendige Wissen über das Funktionieren und Wirken von Versorgungsorganisationen zu generieren, kann und sollte sich die organisationsbezogene Versorgungsforschung prinzipiell aus dem gesamten Methodenrepertoire der qualitativen und quantitativen Sozialforschung gegenstandsangemessen bedienen [21, 22]. Aufgrund der genannten Komplexität in der organisationbezogenen Versorgungsforschung und der dadurch begründeten Relevanz des aktiven Kontextes [4], kommt der Anwendung von Mixed-MethodsAnsätzen eine besondere Bedeutung zu.

\section{Der Mixed-Methods-Ansatz}

Ein Mixed-Methods-Ansatz bedeutet allgemein die Anwendung von qualitativen als auch quantitativen Methoden und unterschei- det sich somit von einem Multimethods-Ansatz, bei dem auch Methoden der gleichen Methodenfamilie angewendet werden [23]. Mixed-Methods-Ansätze können Stärken und Schwächen des jeweils anderen Methodenparadigmas zur Beantwortung einer Forschungsfrage ausgleichen. Aus diesem Grund scheint es sinnvoll, die unterschiedlichen Methoden auch in randomisierten kontrollierten Studien zu kombinieren (für unterschiedliche Möglichkeiten der Kombination mit qualitativen Methoden siehe [24]). Eine auf Praxisanwendung und Verbesserung ausgerichtete organisationsbezogene Versorgungsforschung macht sich Mixed-MethodsAnsätze zu eigen, da diese es ermöglichen, die Organisation sowohl im Sinne des instrumentellen als auch des institutionellen Organisationsbegriffes (vgl. Kap. 1 [2]) zu verstehen und beide Ansätze in Kombination zur Erklärung des Zustandekommens von Patientenoutcomes zu nutzen.

Es liegt eine breite Diskussion zu Designs von Mixed-MethodsStudien vor [25-27]. Für die organisationbezogene Versorgungsforschung sollen an dieser Stelle 2 Haupttypen des Ansatzes vorgestellt werden: das parallele und das sequentielle Design [22, 28, 29]. Das parallele Design bezieht sich auf die gleichzeitige Anwendung quantitativer und qualitativer Methoden zur gleichen Forschungsfrage. Beispielsweise könnte die Mitarbeiterzufriedenheit in einem Krankenhaus mit Fragebögen und gleichzeitig stattfindenden qualitativen Interviews erhoben werden. Bei parallelen Designs besitzt in der Versorgungsforschung oftmals der quantitative Studienzweig Priorität. Die Verknüpfungen beim parallelen Design bestehen zu 2 Zeitpunkten: Bei der Planung der Studie und dem Zusammenführen der Ergebnisse. Dazwischen folgen die 2 Studienzweige den jeweils eigenen Standards und legen die jeweils geltenden Gütekriterien für quantitative oder qualitative Forschung zugrunde. Diese Variante bietet forschungspragmatisch betrachtet die Möglichkeit, dass 2 unterschiedliche Forschungsteams weitgehend unabhängig voneinander arbeiten [23]. Das sequenzielle Design dagegen unterscheidet 2 Phasen, in denen jeweils die Methoden des einen oder des anderen Typs angewandt werden. Charakteristisch für die Durchführung ist hierbei, dass diese Phasen nacheinander erfolgen und die Ergebnisse der ersten Analyse die zweite Studie beeinflussen. Das sequenzielle Design kann in ein Vertiefungsdesign („explanatory“) und ein Vorstudiendesign („exploratory“) unterschieden werden [28]. Das Vertiefungsdesign sieht zuerst eine quantitative und danach eine qualitative Studie vor. Beim Vertiefungsdesign können unklare oder überraschende Ergebnisse einer quantitativen Studie, z. B. aus Patienten- oder Mitarbeiterbefragungen, mittels qualitativen Methoden vertiefend beforscht und erklärt werden [27]. Das Vorstudiendesign dagegen nimmt den umgekehrten Weg: An qualitative Studienergebnisse schließt eine quantitative Studie an. Dieses Design eignet sich u. a. [30] um bspw. Strukturen und Prozesse einer Versorgungsorganisation zu rekonstruieren und davon ausgehend Hypothesen zu generieren, die anschließend mittels einer Mehrebenenanalyse geprüft werden [31]. Mit Mixed-MethodsAnsätzen gehen prinzipiell auch einige methodologische Probleme einher. So gibt es z. B. keinen Konsens über allgemeingültige Gütekriterien [32]. Dies geht eng einher mit der Feststellung, dass es sich bei dem Mixed-Methods-Ansatz um ein „third methodological or research paradigm“ [ 27, S. 318] handelt, das über die Berücksichtigung der jeweils quantitativen und qualitativen Methodologien hinausgeht. Transparenz und Reflexivität des Forschungsprozesses 
sind in diesem Zusammenhang daher essentiell für die Legitimation der generierten Studienergebnisse.

\section{Datenquellen und Datenerhebung}

In der Versorgungsforschung allgemein und in der organisationsbezogenen Versorgungsforschung in besonderer Weise ist darauf zu achten, dass relevante Informationen auf unterschiedlichen Ebenen lokalisiert sein können: Sowohl Daten als auch Interventionen können die Ebene der Patienten und der Professionals ${ }^{2}$ (Mikroebene), der organisationalen Bedingungen (Mesoebene) oder des Gesundheitssystems (Makroebene) betreffen. Für die angemessene Datenerhebung ist es wichtig, ein theoriebasiertes Grundlagenmodell zu definieren [34], aus dem hervorgeht, welche Merkmale (a) der Patienten und Professionals, (b) der Versorgungsstrukturen, (c) der Versorgungsprozesse und (d) des Organisationskontextes zur angemessenen Beschreibung der Versorgungsrealität erhoben werden müssen (siehe Kap. 1 [2]). Merkmale von Versorgungsorganisationen (Mesoebene) ( $\triangleright$ Abb. 1) und Outcomedaten sind zumeist nicht Bestandteil gemeinsamer Datensätze; dazu müssen verschiedene Datenquellen verknüpft werden. Für Fragen der organisationsbezogenen Versorgungsforschung, die Zusammenhänge zwischen Merkmalen von Organisationen und Outcomedaten untersuchen, sollten idealerweise Daten mit einem Mehrebenencharakter vorliegen, $d$. h. hierarchische Datenstrukturen durch die Verknüpfung von Daten auf Mikro- und Mesoebene (eventuell auch auf Makroebene). Nicht selten werden bspw. individuelle patientenbezogene Qualitätsindikatoren oder Outcomes mit Struktur- und Prozessdaten verschiedener Krankenhäusern verknüpft ausgewertet $[35,36]$.

\section{Erhebung von Primärdaten}

Die organisationsbezogene Versorgungsforschung nutzt dazu Primär- ebenso wie Sekundärdaten. Häufig genutzte quantitative Primärdatenerhebungsverfahren in der organisationsbezogenen Versorgungsforschung sind standardisierte Befragungen von Leistungserbringern und Patienten, Dokumentationen der Therapie, aber auch quantifizierende (nicht)teilnehmende Beobachtungen, beispielsweise bei Studien zur Patientensicherheit (z. B. Mitarbeitercompliance von Händedesinfektionsvorschriften). Qualitative Datenerhebungsmethoden sind in der Organisationsforschung von hoher Bedeutung [21, 37-39]. Verwiesen sei hier auf die Diskussionspapiere der Arbeitsgruppe Qualitative Methoden des DNVF, die verschiedene Erhebungsmethoden und Qualitätsstandards diskutieren [40-44]. Zur Analyse der formalen, funktionalen Organisation können bspw. Berichte, Protokolle, Organigramme oder Internetseiten herangezogen werden, die mittels Dokumenten- oder Inhaltsanalyse ausgewertet werden können. Versorgungsorganisationen können aber auch als soziale Orte verstanden werden, an denen sich informelle Regeln und Routinen herausbilden, die nicht zwingend mit der formalen Organisationsbeschreibung einhergehen (vgl. institutioneller Organisationsbegriff Kap. 1 [2]). Zur Rekonstruktion solcher (informaler) Strukturen und Prozesse werden üblicherweise offen gestaltete qualitative Interviews, Gruppenverfahren oder teilnehmende Beobachtungen angewandt. Qualitati-

2 Weitere wichtige an der gesundheitlichen Versorgung Beteiligte sind in Selbsthilfeorganisationen organisierte Personen, die durch ihre eigenen Krankheitserfahrungen in der Regel ein hohes Expertenwissen besitzen [33]. ve Interviews (z. B. Experteninterview, Problemzentriertes Interview) eröffnen über die subjektiven Perspektiven von Mitarbeitenden oder Patienten tiefe Einblicke in die Versorgungsorganisation. Gruppenverfahren (Gruppendiskussion, Fokusgruppe) dagegen eignen sich für die Analyse sozialer Interaktion zwischen Akteuren oder Gruppen, um bspw. das Zustandekommen einer (nicht-rationalen) Entscheidung oder ein typisches Verhalten in einer Versorgungsorganisation zu beleuchten. Sowohl Interviews als auch Gruppenverfahren haben das Ziel, hinter die rationalen Fassaden der Organisation zu blicken und organisationale Handlungen und Entscheidungen verstehend zu erklären (siehe Diskussionspapiere der DNVF-Arbeitsgruppe Qualitative Methoden [40] und Lehrbücher sowie Sammelbände zur allgemeinen Einführung [45-47]).

\section{Erhebung von Sekundärdaten}

Organisationsbezogene Sekundärdatenquellen bezeichnen vorwiegend nicht für Forschungszwecke erhobene Daten. Diese können für Forschungsvorhaben erschlossen werden. Organisationsbezogene Sekundärdaten sind häufig kontinuierlich erhobene und frei verfügbare Sekundärdaten, z. B. die verpflichtenden Qualitätsberichte der Krankenhäuser, das Krankenhausverzeichnis des Statistischen Bundesamtes, Daten des Instituts für das Entgeltsystem im Krankenhaus oder das Arztregister der Kassenärztlichen Bundesvereinigung bzw. Bundesärztekammer. Zudem eignen sich kontinuierlich erhobene, nicht frei verfügbare Daten, wie etwa Daten der externen Qualitätssicherung durch das Institut für Qualitätssicherung und Transparenz im Gesundheitswesen (IQTIG), Leistungsdaten der Krankenkassen, Abrechnungsdaten der Versorgungsorganisationen oder Berichte der Market Access Abteilungen der Industrie. Darüber hinaus können graue Datenquellen von Bedeutung sein, die zu einem speziellen Anlass erhoben wurden und teilweise allgemein und / oder für Forschungszwecke verfügbar sind. Dazu zählen z. B. Erhebungen von Instituten der Kostenträger zu speziellen Fragestellungen, Erhebungen der Berufsverbände zu Patienten- bzw. Anbieterstrukturen (werden häufig nur in Verbandsblättern veröffentlicht), Strukturdaten und Informationen über Spezialisierungen, räumliche Verteilung, erhoben von den Kassenärztlichen Vereinigungen, oder Veröffentlichungen von Selbsthilfeorganisationen auf Basis der Beratungstätigkeit. Zum Umgang mit Sekundärdaten gibt es eine Vielzahl hilfreicher Methodenpapiere [wie bspw. [48-50]].

Für einen umfassenden Überblick zu Datenerhebungsmethoden, frei verfügbaren Daten oder Fragen des Datenschutzes in der Organisationsforschung sei auf Liebig et al. 2017 [51] verwiesen. Primär- und Sekundärdatenquellen, die das Output und Outcome auf der Ebene der Patienten und Professionals (Mikroebene) abbilden, sind demnach bspw. individuelle Abrechnungsdaten der Krankenversicherungen, Registerdaten oder Befragungsdaten. Zum Vergleich von Versorgungsorganisationen sind patientenbezogene Daten auch zur Berücksichtigung des Case-Mix nutzbar oder können als Confounder oder zur Analyse von Subgruppen bei Beobachtungsstudien einfließen.

Grundsätzlich ist bei der Datenerhebung die möglichst präzise Beschreibung und vollzählige Erfassung von Indikatoren zur Abbildung von Versorgungsqualität und den potenziellen (organisationalen) Ursachen von Versorgungsqualität erforderlich, was bei Beobachtungsstudien die Erfassung möglichst aller potentieller Confounder zur Ursachenidentifikation und zur Gewährung eines fairen Vergleichs von Versorgungsorganisationen einschließt. 


\section{Datenanalyse}

\section{Quantitative Methoden der Datenanalyse}

Welche Methodik sich für die Datenanalyse in der organisationsbezogenen Versorgungsforschung eignet, sollte aus der Forschungsfrage, dem Studiendesign sowie den Erhebungsmethoden abgeleitet werden. Während geeignete Datenquellen und -erhebungsmethoden bereits kurz vorgestellt wurden (siehe oben), werden insbesondere für die Evaluation komplexer Interventionen in Organisationen geeignete Studiendesigns in Kapitel 3 [3] näher thematisiert. Für quantitative Datenanalysen in der organisationsbezogenen Versorgungsforschung bestehen, wenn sie der Hypothesenprüfung dienen, die gleichen Anforderungen wie bei anderen konfirmatorischen Prüfungen. Dies hat unmittelbare Konsequenz für die Bestimmung der Stichprobengröße [52], wobei hier neben der Zahl der benötigten Einheiten auf Individualebene ebenso die Zahl der Einheiten auf Organisationsebene zu bestimmen ist. Allerdings sind diesen Anforderungen oftmals „natürliche“ Grenzen gesetzt, die sich aus dem Untersuchungsgegenstand ergeben, etwa wenn zum Organisationsvergleich primär nur eine geringe Zahl von Versorgungsorganisationen zur Verfügung steht. Werden erforderliche Standards nicht erreicht, so müssen bspw. entsprechend Konsequenzen für die Gefahr von Fehlern im Rahmen der statistischen Prüfung (Fehler erster / zweiter Art) durch eine a-posteriori-Poweranalyse bestimmt werden und hinsichtlich inhaltlicher Aspekte kritisch diskutiert werden [53]. Wie oben bereits angedeutet, kommt unter den quantitativen Verfahren für nicht-interventionelle Designs der Mehrebenenanalyse besondere Bedeutung zu, die der spezifischen hierarchischen Datenstruktur der organisationsbezogenen Versorgungsforschung in besonderer Weise gerecht wird, z. B. Patienten (Ebene 1) in Arztpraxen (Ebene 2). Mehrebenenanalysen sind geeignet, Effekte von Organisationsmerkmalen (unter Kontrolle z. B. des Case-Mix) zu identifizieren, was eine ausreichende Tiefe der Daten voraussetzt. Spezifika und Anforderungen dieser Methode wurden bereits an anderer Stelle als Veröffentlichung der Arbeitsgruppe beschrieben [31]. Um die in $>$ Abb. 1 skizzierten Zusammenhänge zu untersuchen, sind neben der Mehrebenenanalyse viele weitere statistische Verfahren geeignet, etwa die üblichen deskriptiven und schließenden statistischen Verfahren ebenso wie strukturanalytische Verfahren wie Strukturgleichungsmodelle, die auch die hierarchische Datenstruktur berücksichtigen können. Diese Verfahren sind, sofern sie - wie häufig der Fall - mit Beobachtungsdaten arbeiten, mit den gleichen Limitationen behaftet wie andere, nicht-interventionelle Studien auch. Insbesondere ist hier die fehlende Möglichkeit zur Untersuchung kausaler Zusammenhänge zu nennen. Verwiesen sei in diesem Zusammenhang auf Shadish et al. [54] und die entsprechende Darstellung in deutscher Sprache von Döring und Bortz [55]. Auf Beobachtungsdaten mit großen Datenmengen (Big Data) basierende Machine Learning Ansätze versuchen Zusammenhangsmuster zu erkennen, können jedoch genauso wenig Kausalität belegen [56]. Sie können potenziell jedoch einen Beitrag zur organisationsbezogenen Versorgungsforschung leisten, indem beispielsweise komplette Versorgungsverläufe über verschiedene an der Versorgung beteiligte Versorgungsorganisationen hinweg analysiert werden können. Interventionellen Studien widmen wir uns im dritten Kapitel [3] detaillierter und betonen an dieser Stelle, dass (cluster-) randomisierte, kontrollierte Studien für den Nach- weis von Wirksamkeit Goldstandard sind, selbst wenn es im Kontext der organisationsbezogenen Versorgungsforschung zumeist größerer Anstrengungen zur Umsetzung bedarf als in der patientenbezogenen Versorgungsforschung. Andererseits tragen konventionelle Studiendesigns wie etwa randomisiert, kontrollierte Studien den Unsicherheiten, Unvorhersehbarkeiten und der Vielfalt interagierender Einflüsse auf ein Outcome in Studien zur Evaluation komplexer Interventionen kaum Rechnung. Die aktuelle internationale Entwicklung in der Versorgungsforschung geht daher dahin, die Komplexität durch den Einbezug von Theorien der Complexity Science stärker zu thematisieren. So sollen Methoden verwendet und entwickelt werden, die pragmatische Aspekte als Elemente einer wissenschaftlich fundierten und belastbaren Herangehensweise berücksichtigen $[57,58]$.

Auswertungen, bei denen Versorgungsprozesse oder Patientenoutcomes zwischen Versorgungsorganisationen verglichen werden, sind eine Kernaufgabe der organisationsbezogenen Versorgungsforschung. Will man Zusammenhänge zwischen Patienten- und Organisationsebene aufzeigen, müssen die erhobenen Patientendaten mit Merkmalen der Versorgungsorganisationen verknüpft werden. Diese Verknüpfbarkeit ist für faire Einrichtungsvergleiche - diez. B. für Standortnachteile kontrollieren - zwar unabdingbar, jedoch wird sie durch schützenswerte Interessen der Professionals und Organisationen eingeschränkt. Hier werden zukünftig erhebliche Anstrengungen und Kooperationen bspw. zwischen Forschung und Kostenträgern nötig sein, um Modelle zur Erklärung der Varianz der Ergebnisqualität zwischen Versorgungsorganisationen zu entwickeln. Hierzu gehören auch vertrauensbildende Maßnahmen mit den betreffenden Einrichtungen. Kann die Fairness der Vergleiche nicht durch eine experimentelle Grundanlage der Studie gewährleistet werden, so sollten Techniken zum Einsatz kommen, die systematische initiale beobachtete Gruppendifferenzen statistisch kontrollieren [59] (insbesondere [mehrebenen]regressionsanalytische Verfahren, propensity score gestützte Verfahren, oder stratifizierte Analysen).

\section{Qualitative Methoden der Datenanalyse}

Qualitative Methoden spielen in der organisationsbezogenen Versorgungsforschung wie schon beschrieben eine bedeutende Rolle. Die Auswertung der beschriebenen Formen der qualitativen Datenerhebung erfolgt mittels einer regelgeleiteten Interpretation der Daten, die üblicherweise als wortgetreu verschriftlichte Transkripte oder Protokolle vorliegen. Hierzu werden unterschiedliche Auswertungsverfahren angewandt $[45,46]$. Es kann unterschieden werden zwischen (1) kategorisierenden Verfahren wie bspw. der qualitativen Inhaltsanalyse $[29,60]$ oder dem theoretischen Codieren zur gegenstandsbezogenen Theoriebildung (Grounded Theory) [61] und (2) sequenziellen Verfahren wie bspw. der Konversationsanalyse [62]. Da sich letztere Verfahren streng an der Struktur des Einzelfalls orientieren, kommen in der organisationsbezogenen Versorgungsforschung eher die kategorisierenden Verfahren zur Anwendung. In diesem Zuge zeigt sich eine methodologische Lücke: die rekonstruktive Sozialforschung findet derzeit wenig Beachtung, obwohl sie sich für etliche Fragen der organisationsbezogenen Versorgungforschung besonders gut eignet (z. B. zur Rekonstruktion von informeller Organisation als Reaktion auf veränderte Rahmenbedingungen auf der Makroebene, zur Analyse von Verän- 
derungen im Management eines Krankenhauses und den Auswirkungen auf die Organisationskultur). Rekonstruktive Sozialforschung zielt darauf ab, die von den Personen nicht explizierbaren und somit latenten Sinnstrukturen, z. B. Handlungs- und Deutungsmuster, aus objektiviertem Material (z. B. Interviewtranskripte) interpretativ abzuleiten. Eine Einführung in die rekonstruktive Sozialforschung, die bspw. die objektive Hermeneutik [63], die dokumentarische Methode [64] oder die Konversationsanalyse [65] umfasst, ist in der Literatur zu finden $[18,19]$. Qualitative Datenanalysen können in der organisationsbezogenen Versorgungsforschung insbesondere dann angewendet werden, wenn es darum geht, Organisationsstrukturen, -prozesse und -kulturen innerhalb von Versorgungsorganisationen zu verstehen und deren Beitrag für das Zustandekommen eines Versorgungsoutcomes zu erklären.

\section{Gütekriterien}

Gütekriterien für die Datenqualität, Datenerhebung, Datenanalyse und das Berichten von Ergebnissen unterscheiden sich nicht von anderen in der Versorgungsforschung und Epidemiologie. Verwiesen sei zunächst auf einschlägige Reporting Guidelines: für Beobachtungsstudien können die STROBE-Kriterien [66], für experimentelle Studien die CONSORT- und TIDieR-Kriterien, für Studien zur Qualitätsverbesserung die SQUIRE-Kriterien als zentrale Orientierung gelten [67-70]. Für qualitative Studien ist die COREQ Reporting Guideline zu beachten [71]. Zur Qualitätsbewertung qualitativer Versorgungsforschungsstudien sei auf die Veröffentlichung der DNVF-AG Qualitative Methoden verwiesen [43]. May et al. [72] und Pfadenhauer et al. [73] geben zudem einen Rahmen für qualitativ hochwertige Implementationsstudien. Eine für die organisationsbezogene Versorgungsforschung spezifische Reporting Guideline oder ein entsprechendes Amendement zu einer existierenden Reporting Guideline liegen nach unserer Kenntnis bislang nicht vor.

\section{FAZIT}

In der Versorgungsforschung ist die Sicht auf organisationale Zusammenhänge bisher vielfach noch versperrt durch die Unkenntnis geeigneter theoretischer und methodischer Ansätze. So wird der Organisationsbezug von Forschungsfragen und -daten häufig nicht gesehen und die Potenziale der organisationsbezogenen Versorgungsforschung bleiben dadurch oft ungenutzt. Durch die Ergänzung der bisher meist outcomebezogenen Zielgrößen in der Versorgungsforschung um Zielgrößen mit Fokus auf Versorgungsstrukturen, -prozesse und -kulturen in Versorgungsorganisationen ist die organisationsbezogene Versorgungsforschung in der Lage, die vielfach beschriebene Black Box zur Erklärung der Variation von Patientenoutcomes zwischen Versorgungsorganisationen oder zur Erklärung der Wirkmechanismen von Interventionen in der Versorgung zu beleuchten. Um dies jedoch leisten zu können, ist die Nutzung geeigneter Datenquellen sowie die Anwendung und Integration geeigneter Methoden der Datenerhebung, -nutzung und -analyse erforderlich und für die Zukunft essentiell.

\section{Interessenkonflikt}

Die Autoren geben an, dass kein Interessenkonflikt besteht.

\section{Literatur}

[1] Pfaff H, Albert U-S, Bornemann R et al. Methoden für die organisationsbezogene Versorgungsforschung. Gesundheitswesen 2009; 71: 777-790

[2] Ansmann L, Baumann W, Gostomzyk J et al. DNVF-Memorandum III Methoden für die Versorgungsforschung, Teil 4 - Konzept und Methoden der organisationsbezogenen Versorgungsforschung, Kapitel 1 - Definition und Konzept der organisationsbezogenen Versorgungsforschung. Gesundheitswesen 2019. DOI 10.1055/a-0862-0527

[3] Wirtz MA, Bitzer EM, Albert US et al. DNVF-Memorandum III - Methoden für die Versorgungsforschung, Teil 4 - Konzept und Methoden der organisationsbezogenen Versorgungsforschung, Kapitel 3 - Methodische Ansätze zur Evaluation und Implementierung komplexer Interventionen in Versorgungsorganisationen. Gesundheitswesen 2019. DOI 10.1055/a-0862-0588

[4] Schrappe M, Pfaff H. Versorgungsforschung vor neuen Herausforderungen. Konsequenzen für Definition und Konzept. Gesundheitswesen 2016; 78: 689-694

[5] Institut für Qualität und Wirtschaftlichkeit im Gesundheitswesen. Allgemeine Methoden. Online https://www.iqwig.de/de/methoden/ methodenpapier.3020.html

[6] Troidl H, Wechsler AS, McKneally MF, How to choose a relevant endpoint.In Troidl $\mathrm{H}$ et al., (Hrsg.). Surgical research. Basic principles and clinical practice. 3. Aufl. New York: Springer; 1998: 303-319

[7] Geraedts M, Drösler SE, Döbler K et al. DNVF-Memorandum III „Methoden für die Versorgungsforschung", Teil 3. Methoden der Qualitäts- und Patientensicherheitsforschung. Gesundheitswesen 2017; 79: 883-886

[8] Körner M, Ansmann L, Schwarz B et al., (Hrsg.). Organizational Behaviour in Healthcare. Theoretical Approaches, Methods and Empirical Results. 1. Aufl. Wien, Österreich: LIT Verlag; 2018

[9] Simon M. Das Gesundheitssystem in Deutschland. Eine Einführung in Struktur und Funktionsweise. 6. Aufl. Bern: Hogrefe: 2017

[10] Arrow KJ, Arrow In Finkelstein A, Arrow KJ et al., (Hrsg.). Moral hazard in health insurance. [developments since Arrow (1963)]. New York, NY: Columbia Univ. Press; 1963: 2015: 81-136

[11] Kühl S. Organisationen. Eine sehr kurze Einführung. 1. Aufl. Wiesbaden: VS Verl. für Sozialwiss; 2011

[12] Scott T, Mannion R, Davies H et al. The Quantitative Measurement of Organizational Culture in Health Care. A Review of the Available Instruments. Health Serv Res 2003; 38: 923-945

[13] Schein EH. Organizational culture and leadership. 4. Aufl. San Francisco CA: Jossey-Bass; 2010

[14] Stricoff RS. Understanding safety's role in culture and climate. Occupational Hazards 2005; 67: 25-27

[15] Hammer A. Zur Messung von Sicherheitskultur in deutschen Krankenhäusern. Köln: Universität zu Köln; 2012

[16] Jung T, Scott T, Davies HTO et al. Instruments for Exploring Organizational Culture: A Review of the Literature. Public Administration Review 2009; 69: 1087-1096

[17] Yauch CA, Steudel HJ. Complementary Use of Qualitative and Quantitative Cultural Assessment Methods. Organizational Research Methods 2003; 6: 465-481

[18] Strübing J. Qualitative Sozialforschung. Eine Komprimierte Einführung Für Studierende. 2. Aufl. Berlin/Boston: Walter de Gruyter GmbH; 2018 
[19] Przyborski A, Wohlrab-Sahr M. Qualitative Sozialforschung. Ein Arbeitsbuch. 4. Aufl. München: Oldenbourg; 2014

[20] Berwick DM. The science of improvement. JAMA 2008; 299: 1182-1184

[21] Kühl S, Strodtholz P, Taffertshofer A. Qualitative und quantitative Methoden der Organisationsforschung - ein Überblick. In Kühl S, (Hrsg.). Handbuch Methoden der Organisationsforschung. Quantitative und qualitative Methoden. 1. Aufl. Wiesbaden: VS Verlag für Sozialwissenschaften; 2009: 13-27

[22] Swanson RA, Holton EF., (Hrsg.). Research in organizations. Foundations and methods of inquiry. San Francisco, CA: Berrett-Koehler; 2005

[23] Kuckartz U. Mixed Methods. Methodologie, Forschungsdesigns und Analyseverfahren. Wiesbaden: Springer-Verlag; 2014

[24] Lewin S, Glenton C, Oxman AD. Use of qualitative methods alongside randomised controlled trials of complex healthcare interventions. Methodological study. BMJ (Clinical research (ed.). 2009: 339: b3496

[25] Johnson RB, Onwuegbuzie A]. Mixed Methods Research. A Research Paradigm Whose Time Has Come. Educational Researcher 2004; 33 : $14-26$

[26] Creswell JW, Plano Clark VL. Choosing a mixed methods design. Designing and conducting mixed methods research 2011; 53-106

[27] Tashakkori A, Teddlie C., (Hrsg.). Sage handbook of mixed methods in social \& behavioral research. 2. Aufl. Los Angeles: Calif.: SAGE; 2010

[28] Creswell JW. A concise introduction to mixed methods research. Los Angeles: SAGE Publications; 2014

[29] Mayring P. Qualitative Inhaltsanalyse. Grundlagen und Techniken. 12. Aufl. Weinheim/Basel: Beltz; 2015

[30] Noest S, Ludt S, Klingenberg A et al. Involving patients in detecting quality gaps in a fragmented healthcare system. Development of a questionnaire for Patients' Experiences Across Health Care Sectors (PEACS). International journal for quality in health care : journal of the International Society for Quality in Health Care 2014; 26: 240-249

[31] Ansmann L, Kuhr K, Kowalski C. Mehrebenenanalysen in der organisationsbezogenen Versorgungsforschung - Nutzen, Voraussetzungen und Durchführung. Das Gesundheitswesen 2016; 79: 203-209

[32] Morgan DL. Paradigms Lost and Pragmatism Regained. Journal of Mixed Methods Research 2007; 1: 48-76

[33] Borgetto B. Selbsthilfezusammenschlüsse. In Pfaff H, Neugebauer E, Glaeske G, (Hrsg.). Lehrbuch Versorgungsforschung. Systematik - Methodik - Anwendung. 2. Aufl. Stuttgart: Schattauer GmbH; 2017: 187-196

[34] Baumann W, Farin E, Menzel-Begemann A et al. Memorandum IV. Theoretische und normative Fundierung der Versorgungsforschung. Gesundheitswesen 2016; 78: 337-352

[35] Cho S-H. Using multilevel analysis in patient and organizational outcomes research. Nursing research 2003; 52: 61-65

[36] Sjetne IS, Veenstra M, Stavem K. The effect of hospital size and teaching status on patient experiences with hospital care: a multilevel analysis. Medical care 2007; 45: 252-258

[37] Cohen DJ, Crabtree BF. Evaluative criteria for qualitative research in health care. Controversies and recommendations. Annals of family medicine 2008; 6: 331-339

[38] Mays N, Pope C. Qualitative research in health care. Assessing quality in qualitative research. BMJ (Clinical research (ed.). 2000: 320: 50-52

[39] Ernst S. Prozessorientierte Methoden in der Arbeits- und Organisationsforschung. Eine Einführung. Wiesbaden: VS Verlag für Sozialwissenschaften / GWV Fachverlage GmbH Wiesbaden; 2010

[40] Karbach U, Stamer M, Holmberg C et al. Qualitative Studien in der Versorgungsforschung - Diskussionspapier, Teil 2. Stand qualitativer Versorgungsforschung in Deutschland - ein exemplarischer Überblick. Gesundheitswesen. Das Gesundheitswesen 2012; 08/09: 516-525
[41] Pohontsch NJ, Müller V, Brandner S et al. Gruppendiskussionen in der Versorgungsforschung - Teil 1. Einführung und Überlegungen zur Methodenwahl und Planung. Gesundheitswesen 2017

[42] Meyer T, Karbach U, Holmberg C et al. Qualitative Studien in der Versorgungsforschung - Diskussionspapier, Teil 1: Gegenstandsbestimmung. Gesundheitswesen 2012; 74: 510-515

[43] Stamer M, Güthlin C, Holmberg C et al. Qualitative Studien in der Versorgungsforschung - Diskussionspapier, Teil 3: Qualität qualitativer Studien. Gesundheitswesen 2015; 77: 966-975

[44] Karbach U, Stamer M, Holmberg C et al. Qualitative Studien in der Versorgungsforschung - Diskussionspapier, Teil 2: Stand qualitativer Versorgungsforschung in Deutschland - ein exemplarischer Überblick. Gesundheitswesen 2012; 74: 516-525

[45] Lamnek S, Krell C. Qualitative Sozialforschung. 6. Aufl. Weinheim/ Basel: Beltz; 2016

[46] Flick U. Qualitative Sozialforschung. Eine Einführung. 7. Aufl. Reinbek bei Hamburg: Rowohlt Taschenbuch Verlag; 2016

[47] Kühl S. (Hrsg.). Handbuch Methoden der Organisationsforschung. Quantitative und qualitative Methoden. 1. Aufl. Wiesbaden: VS Verlag für Sozialwissenschaften; 2009

[48] Neubauer S, Zeidler J, Lange A et al. Grundlagen und Methoden von GKV-Routinedatenstudien. Online http://diskussionspapiere.wiwi. uni-hannover.de/pdf_bib/dp-534.pdf

[49] Swart E, Gothe H, Geyer S et al. Gute Praxis Sekundärdatenanalyse (GPS). Leitlinien und Empfehlungen. Gesundheitswesen 2015; 77: $120-126$

[50] Swart E, Ihle P, Gothe $\mathrm{H}$ et al. Routinedaten im Gesundheitswesen. Handbuch Sekundärdatenanalyse: Grundlagen, Methoden, und Perspektiven. 2. Aufls.l.: Verlag Hans Huber; 2014

[51] Liebig S, Matiaske W, Rosenbohm S. (Hrsg.). Handbuch Empirische Organisationsforschung. Wiesbaden: Springer Fachmedien Wiesbaden 2017

[52] Maas CJM, Hox JJ. Sufficient Sample Sizes for Multilevel Modeling. Methodology 2005; 1: 86-92

[53] Gillett R. Post hoc power analysis. Journal of Applied Psychology 1994; 79: 783-785

[54] Shadish WR, Cook TD, Campbell DT. Experimental and quasi-experimental designs for generalized causal inference. Boston, New York: Houghton Mifflin; 2002

[55] Bortz ], Döring N. Forschungsmethoden und Evaluation. Für Human- und Sozialwissenschaftler. 4. Aufl. Berlin Heidelberg: SpringerMedizin-Verl; 2006

[56] Wegscheider K, Koch-Gromus U. Big Data Analytics. In Pfaff H, Neugebauer E, Glaeske G, (Hrsg.). Lehrbuch Versorgungsforschung. Systematik - Methodik - Anwendung. 2. Aufl. Stuttgart: Schattauer GmbH; 2017: 134-139

[57] Greenhalgh T, Papoutsi C. Studying complexity in health services research: desperately seeking an overdue paradigm shift. BMC medicine 2018; 16: 95

[58] Braithwaite J, Churruca K, Long JC et al. When complexity science meets implementation science: a theoretical and empirical analysis of systems change. BMC medicine 2018; 16: 63

[59] Kuss O, Blettner M, Börgermann J. Propensity Score. An Alternative Method of Analyzing Treatment Effects. Deutsches Arzteblatt international 2016; 113: 597-603

[60] Kuckartz U. Qualitative Inhaltsanalyse. Methoden, Praxis, Computerunterstützung. 3. Aufl. Weinheim/Basel: Beltz Juventa; 2016

[61] Corbin JM, Strauss AL. Basics of Qualitative Research. Techniques and Procedures for Developing Grounded Theory. 4. Aufl. Los Angeles Calif. u. a.: SAGE;: 2015 
[62] Forsthoffer I, Dittmar N. Konversationsanalyse. In Kühl S, (Hrsg.). Handbuch Methoden der Organisationsforschung. Quantitative und qualitative Methoden. 1. Aufl. Wiesbaden: VS Verlag für Sozialwissenschaften; 2009: 348-368

[63] Wernet A. Einführung in die Interpretationstechnik der Objektiven Hermeneutik. 3. Aufl. Wiesbaden: VS Verlag für Sozialwissenschaften / Springer Fachmedien Wiesbaden GmbH Wiesbaden; 2009

[64] Bohnsack R, Nentwig-Gesemann I, Nohl A-M., (Hrsg.). Die dokumentarische Methode und ihre Forschungspraxis. Grundlagen qualitativer Sozialforschung. 3. Aufl. Wiesbaden: Springer VS; 2013

[65] Sidnell J, Stivers T., (Hrsg.). The handbook of conversation analysis. Malden, MA, Oxford, Chichester, West Sussex, UK: Wiley Blackwell; 2014

[66] von Elm E, Altman DG, Egger M et al. Das Strengthening the Reporting of Observational Studies in Epidemiology (STROBE-) Statement. Der Internist 2008; 49: 688-693

[67] Campbell MK, Piaggio G, Elbourne DR et al. Consort 2010 statement. Extension to cluster randomised trials. British Medical Journal 2012; 345: e5661

[68] Schulz KF, Altman DG, Moher D. CONSORT 2010 Statement. Updated guidelines for reporting parallel group randomised trials. BMC medicine 2010; 8: 18

[69] Hoffmann TC, Glasziou PP, Boutron I et al. Better reporting of interventions. Template for intervention description and replication (TIDieR) checklist and guide. BMJ (Clinical research (ed.). 2014: 348: g1687

[70] Ogrinc G, Mooney SE, Estrada C et al. The SQUIRE (Standards for QUality Improvement Reporting Excellence) guidelines for quality improvement reporting: explanation and elaboration. Quality \& safety in health care 2008; 17 (Suppl 1): i13-i32

[71] Tong A, Sainsbury P, Craig J. Consolidated criteria for reporting qualitative research (COREQ). A 32-item checklist for interviews and focus groups. International journal for quality in health care : journal of the International Society for Quality in Health Care 2007; 19: 349-357

[72] May C, Finch T, Mair F et al. Understanding the implementation of complex interventions in health care. The normalization process model. BMC health services research 2007; 7: 148

[73] Pfadenhauer LM, Gerhardus A, Mozygemba K et al. Making sense of complexity in context and implementation. The Context and Implementation of Complex Interventions (CICl) framework. Implementation science: IS 2017; 12: 21

\section{Entwicklung, Kommentierung und Abstimmung des Memorandums}

Nach den Standards der Erstellung von Memoranden des DNVF wurde die Zustimmung des DNVF-Vorstands zur Erarbeitung eines Memorandums eingeholt und alle Mitglieder und Mitgliedsorganisationen wurden zur Mitarbeit eingeladen. Es haben sich 20 Personen aktiv an der Erstellung des Memorandums beteiligt (Autorengruppe). Die Autorengruppe stimmte die Grundstruktur des Memorandums ab, bildete für jedes der 3 Kapitel eine Autorengruppe und erstellte gemeinsam den Entwurf des Memorandums. Struktur und Inhalt wurden auf dem Deutschen Kongress für Versorgungsforschung in 2017 und 2018 diskutiert. Alle Mitglieder- und Mitgliedsorganisationen des DNVF hatten in einer 3-monatigen Kommentierungsphase vom 10.07. bis 10.10.2018 die Möglichkeit, zum Entwurf Stellung zu nehmen. Auf die Kommentierungen wurde in einer finalen Überarbeitung vollumfänglich reagiert. Danach wurde eine finale Fassung erstellt, die an die ordentlichen sowie an die korrespondierenden institutionellen Mitglieder des DNVF mit der Einladung zur Mitzeichnung versandt wurde. Die Autoren des aktuellen Memorandums möchten sich bei allen am Konsentierungsprozess Beteiligten für ihre konstruktiven Diskussionsbeiträge bedanken.

Folgende Fachgesellschaften haben sich im Rahmen des Kommentierungsprozesses am Memorandum zu Konzept und Methoden der organisationsbezogenen Versorgungsforschung beteiligt:

- Deutsche Gesellschaft für Gynäkologie und Geburtshilfe

- Deutsche Gesellschaft für Pflegewissenschaft

- Deutsche Gesellschaft für Prävention und Rehabilitation von Herz-Kreislauferkrankungen

- Deutsche Gesellschaft für Rehabilitationswissenschaft

- Deutsche Gesellschaft für Senologie

Folgende Wissenschaftliche Institute und Forschungsverbünde haben sich im Rahmen des Kommentierungsprozesses am Memorandum zu Konzept und Methoden der organisationsbezogenen Versorgungsforschung beteiligt:

- Centre for Health and Society

- LVR-Institut für Versorgungsforschung

Folgende persönliche Mitglieder des DNVF haben sich im Rahmen des Kommentierungsprozesses am Memorandum zu Konzept und Methoden der organisationsbezogenen Versorgungsforschung beteiligt:

- Eugenia Larjow

Das Memorandum zu Konzept und Methoden der organisationsbezogenen Versorgungsforschung wird von folgenden ordentlichen und korrespondierenden institutionellen Mitgliedern des Deutschen Netzwerks Versorgungsforschung e. V. getragen.

Von folgenden Mitgliedern der Sektion „Fachgesellschaften“:

- Deutsche Diabetes Gesellschaft e.V.

- Deutsche Gesellschaft für Allgemeinmedizin

- Deutsche Gesellschaft für Ambulante Allgemeine Pädiatrie

- Deutsche Gesellschaft für Anästhesiologie und Intensivmedizin e.V. 
- Deutsche Gesellschaft für Angiologie - Gesellschaft für Gefäßmedizin e.V.

- Deutsche Gesellschaft für Arbeitsmedizin und Umweltmedizin e.V.

- Deutsche Gesellschaft für Gynäkologie und Geburtshilfe e.V.

- Deutsche Gesellschaft für Hämatologie und Medizinische Onkologie e.V.

- Deutsche Gesellschaft für Innere Medizin e.V.

- Deutsche Gesellschaft für Kardiologie - Herz- und Kreislaufforschung e.V.

- Deutsche Gesellschaft für Kinder- und Jugendmedizin e.V.

- Deutsche Krebsgesellschaft e.V.

- Deutsche Gesellschaft für Medizinische Soziologie e.V.

- Deutsche Gesellschaft für Mund-, Kiefer und Gesichtschirurgie

- Deutsche Gesellschaft für Neurochirurgie e.V.

- Deutsche Gesellschaft für Neurologie e.V.

- Deutsche Gesellschaft für Orthopädie und Orthopädische Chirurgie e.V.

- Deutsche Gesellschaft für Psychiatrie und Psychotherapie, Psychosomatik und Nervenheilkunde e.V.

- Deutsche Gesellschaft für Rehabilitationswissenschaften e.V.

- Deutsche Gesellschaft für Schlafforschung und Schlafmedizin e.V.

- Deutsche Gesellschaft für Senologie e.V.

- Deutsche Gesellschaft für Sozialmedizin und Prävention e.V.

- Deutsche Gesellschaft für Zahn-, Mund- und Kieferheilkunde e.V.

- Deutsche Ophtalmologische Gesellschaft e.V.

- Deutsche Sepsis-Gesellschaft e.V.

Von folgenden Mitgliedern der Sektion „Wissenschaftliche Institute und Forschungsverbände":

- Centre for Health and Society, Universitätsklinikum Düsseldorf
- Center for Health Care Research, Universitätsklinikum Hamburg-Eppendorf

- Gesundheit Österreich GmbH

- Institut für Hausarztmedizin der Universität Bonn

- Institut für Medizinsoziologie, Versorgungsforschung und Rehabilitationswissenschaft der Humanwissenschaftlichen Fakultät und der Medizinischen Fakultät der Universität zu Köln (KöR)

- Institut für Patientensicherheit, Universitätsklinikum Bonn

- Interdisziplinäres Zentrum für Versorgungsforschung im Gesundheitswesen der Universität Witten/Herdecke

- Landesinstitut Gesundheit des Bayerischen Landesamts für Gesundheit und Lebensmittelsicherheit

- LVR-Institut für Versorgungsforschung

- Medizinische Hochschule Brandenburg, Theodor Fontane

- Sektion Versorgungsforschung und Rehabilitationsforschung, Universitätsklinikum Freiburg

- Wissenschaftliches Institut der Niedergelassenen Hämatologen und Onkologen $\mathrm{GmbH}$

- Zentrum für Evidenzbasierte Gesundheitsversorgung, Universitätsklinikum Carl Gustav Carus an der TU Dresden

- Zentrum für Versorgungsforschung Köln

- Universität Potsdam, Professur für Rehabilitationswissenschaften

- Von folgenden Mitgliedern der Sektion „Juristische Personen und Personenvereinigungen“:

- Berufsverband der Kinder- und Jugendärzte e.V.

- Deutscher Verband für Gesundheitssport und Sporttherapie e.V.

- Kassenärztliche Bundesvereinigung

- OptiMedis AG

- 4QD - Qualitätskliniken.de GmbH

\section{Hinweis}

Dieser Artikel wurde gemäß des Erratums vom 24.4.2019 geändert.

\section{Erratum}

Im oben genannten Artikel war in der html-Version bei einer Autorin die technische Zuordnung des zweiten Instituts falsch. Korrekt ist:

\section{Lena Ansmann}

1 Fakultät für Medizin und Gesundheitswissenschaften, Department für Versorgungsforschung, Carl von Ossietzky Universität Oldenburg, Oldenburg

3 Humanwissenschaftliche Fakultät und Medizinische Fakultät, Institut für Medizinsoziologie, Versorgungsforschung und Rehabilitationswissenschaften, Universität zu Köln, Köln 